\title{
Spontaneous Intestinal Perforation and Infantile Hypertrophic Pyloric Stenosis in a Preterm Neonate: A Linked Etiopathogenesis?
}

\author{
Amitoj Singh Chhina ${ }^{1}$ (D) Mohit Singhal ${ }^{1}$. \\ Malathi Raja ${ }^{1}$ - Arvind Shenoi ${ }^{1}$
}

Received: 2 April 2015 / Accepted: 26 May 2015 / Published online: 10 June 2015

(C) Dr. K C Chaudhuri Foundation 2015

To the Editor: A 29-wk, $1020 \mathrm{~g}$ preterm baby boy was born by cesarean section to a 30-y-old G2A1 with placental abruption and an otherwise normal antenatal course. Trophic feeds were started on day 3 of life. Intestinal perforation was suspected on day 6 and confirmed by exploratory laparotomy. An ileostomy was created. Histopathological examination revealed normal intestinal tissue ruling out necrotizing enterocolitis. Feeds were reintroduced on day 12 and full feeds were reached within a week. On day 40, the baby developed non-bilious vomiting and significant residuals. Prokinetic agents, and later, continuous feeds were started. Intermittent vomiting continued. On day 72 , the baby had apneic episodes and feeds were stopped. The clinical examination was normal apart from mild abdominal distension. Arterial blood gas showed hypochloremic metabolic alkalosis; abdominal ultrasound revealed a hypertrophic pylorus with $14.9 \mathrm{~mm}$ length and $4.8 \mathrm{~mm}$ mural thickness. On day 76, pyloromyotomy was performed after confirming pyloric hypertrophy intraoperatively. Oral feeds were started on day 77 and breastfeeding on day 81 . There was no further feeding intolerance or intractable nonbilious vomiting and the baby is thriving well.

In spontaneous intestinal perforation (SIP), the histopathology shows focal perforations, usually in the ileum, and muscularis externa necrosis without any ischemic damage $[1,2]$. Animal models suggest that impairments in signaling pathways like nitric oxide synthases (NOS), insulin-like growth factors, and epidermal growth factors may lead to ileal

Amitoj Singh Chhina

amitoj13@gmail.com

1 Department of Pediatrics and Neonatology, Cloudnine Hospital, Old Airport Road, Kodihalli, Murugeshpalya, Bangalore 560017, India submucosal thinning with accompanying mucosal hyperplasia [2]. Global depletion of NOS, and therefore, nitric oxide, which modulates gastrointestinal smooth muscle relaxation, leads to impaired intestinal motility and reduced transforming growth factor-alpha in the muscularis externa, making the distal intestine susceptible to perforation $[1,2]$.

Animal studies have also illuminated the role of NOS in the etiopathogenesis of infantile hypertrophic pyloric stenosis (IHPS). Mice with reduced NOS activity developed grossly enlarged stomachs with hypertrophy of the circular muscle layer [3]. Endothelial NOS-knockout mice treated with dexamethasone were found to have a higher risk of developing both acute pyloric stenosis and ileal perforation [1].

This indicates a shared etiopathogenesis between the two conditions, likely due to aberrant NOS function. Thus, it may be worth keeping in mind the possiblility of IHPS in preterm neonates who have already had SIP, especially with symptoms resembling feeding intolerance.

Conflict of Interest None.

Source of Funding None.

\section{References}

1. Gordon PV, Herman AC, Marcinkiewicz M, Gaston BM, Laubach VE, Aschner JL. A neonatal mouse model of intestinal perforation: investigating the harmful synergism between glucocorticoids and indomethacin. J Pediatr Gastroenterol Nutr. 2007;45:509-19.

2. Gordon PV. Understanding intestinal vulnerability to perforation in the extremely low birth weight infant. Pediatr Res. 2009;65: $138-44$.

3. Huang PL, Dawson TM, Bredt DS, Snyder SH, Fishman MC. Targeted disruption of the neuronal nitric oxide synthase gene. Cell. 1993;75:1273-86. 